\title{
A Novel and Efficient Algorithm for Deploying Mobile Sensors in Subsurface
}

\author{
Dimple Juneja \\ MM Institute of Computer Technology \& Business Management, MM University \\ Mullana (Ambala), Haryana, India \\ E-mail: dimplejunejagupta@gmail.com \\ Atul Sharma \\ MM Institute of Computer Technology \& Business Management, MM University \\ Mullana (Ambala), Haryana, India \\ E-mail: atul44885@gmail.com \\ Punit Kumar \\ Department of Mechanical Engineering, NIT, Kurukshetra, Haryana, India
}

S.S. Iyengar

Dept. of Computer Science, Louisiana State University, USA

A.K Sharma

YMCA Institute of Engineering, Faridabad, Haryana, India

\begin{abstract}
The demand for oil is growing steadily from emerging and developing economies while oil field discoveries continue to decline. Therefore the gap between demand and supply will increase with time. Subsurface Exploration deals with extracting valuable hydrocarbons from oil wells. Due to its hazardous nature, it's one of the most difficult fields to carry experimentations on. Uncertainties associated with this field are result of various factors such as lack of information regarding location, size and spread of natural resource. In order to handle above listed factors, mobile wireless sensors seems to be a promising paradigm for increasing productivity and throughput by serving as intelligent investigators. At the time of this listing, none of the researchers have proposed the deployment of mobile wireless sensors in the oil fields. Therefore, this work contributes a unique strategy that could deploy mobile sensors in the subsurface so as to get real time information which otherwise is not possible. Moreover, the proposed algorithm provides efficient coverage and connectivity metric. Also, a mathematical model has been presented along with its comparison with other existing node placement strategies in other related fields and it is found that our algorithm provides better coverage and connectivity.
\end{abstract}

Keywords: Wireless sensor networks, Subsurface exploration, Sensor deployment

\section{Introduction}

Subsurface exploration [Juneja, Dimple. et al., (2009)] is the process of extracting hydrocarbons available beneath earth crust. The figures [CIA] presented by Central Intelligence Authority (CIA) reflects the facts that increase in demand of hydrocarbons is directly proportional to the consumption of hydrocarbons. The available facts [AEC (2008)] indicate that only 30\% (approximately) of oil is recovered from an available field \& rest of $70 \%$ remains un-extracted due to lack of information or various other factors, such as amount remaining, pressure, temperature \& other real time conditions. To keep pace with increased demands as well as consumption, oil companies are in never ending pursuit for improving the production of existing wells with the help of latest technologies. In fact available literature [Roger M. et al., (2004), Colibrys, CNBC] reveals the fact that very few researchers have spent little time looking at the more recent work from other fields. One example of this is the lack of wireless sensor networks [David Culler, et al., (2004), WSN] within the community of subsurface exploration. There are many properties of sensors such as intelligent sensing, collection and processing of data, 
make them well suited to uncertain environments. The adoption of Mobile Sensors as architectural elements enables us to achieve the desired delegated goal of enhanced oil recovery which in turn demands the formulation and development of a novel sensor deployment strategy in subsurfaces.

Usually, static sensors [Eisenman, S. B., et al., (2006), Ghosh, A. (2004)] are being used, which do not have any kind of locomotive capability, whereas mobile sensors may be deployed for better performance [B. Liu, et al., (2005)]. A mobile sensor network [Ghosh, A. (2004), X. Du, et al., (2005)] comprises of a distributed collection of nodes, which in addition to sensing, computation and communication abilities, is also possessed with locomotion capabilities. Deploying mobile sensors result in increased network coverage, better routing performance, self deployment and self reconfiguration. This research proposes deployment of mobile sensor in subsurfaces since reconfiguration of nodes depending on real time conditions shall be far more easy and attainable.

The paper is structured as follows: Section II discusses the motivation of deploying mobile sensors in oil fields. Section III throws light on the light of work of researchers who proposed deployment strategies for wireless sensors in other related fields Section IV presents the proposed strategy along with its pros and cons. Also comparison of our strategy with others is being presented. Finally, section V concludes the paper and illustrates the future work.

\section{Motivation of Deploying Mobile Sensors in Oil Fields}

Advances in Micro-Mechanical Systems (MEMS) have allowed integration of sensors, actuators, mechanical elements and wireless communication on a common silicon substrate with the help of micro-fabrication. These integrated units are low-cost and small form-factor embedded systems called sensor nodes [Sohrabi, K. . et al., (2000), Estrin, D. , et al., (1999)]. Wireless sensors leading to a robust and scalable WSN are being successfully used for energy management [Bin Lu, et al., (2005)], medical monitoring [Virone, G. , et al., (2006)], battlefield management / surveillance [Tatiana Bokareva, et al., (2006)], habitat monitoring [Alan Mainwaring, et al., (2002)], and security systems [Nils Timotheus, et al., (2008)] and so on. Although the proposals [AEC (2008)] have been invited for the deployment of sensors in oil fields but none have succeeded in deployment of sensors in subsurface exploration. The need for self-effacing and remote monitoring is the main motivation for deploying a sensing and communication network consisting of a large number of these battery powered sensor nodes.

\section{Background}

Traditionally in WSN network application, sensors are deployed with aim of monitoring a specified area. In certain applications it was always easy to select sites where sensors could have been placed, e.g. sensors placed in a building for firefighting [Sammarco, J.J., et al., (2007)] purpose or to monitor intrusion detection and such a deployment is termed as deterministic deployment. But in non-deterministic deployment like habitat monitoring [Alan Mainwaring, et al., (2002)] in hostile locations, large numbers of sensors are simply scattered via air drop with the hope that they connect with each other and start passing data within themselves. For both strategies, various, algorithms [Estrin, D. , et al., (2001), Akkaya, K. ., et al., (2005), Wang, Y.C. , et al., (2005)] have been developed ensuring the coverage [Huang, C.-F., et al., (2003), Bai, X., et al., (2006)] and connectivity [Bai, X., et al., (2006), Wang, X. , et al., (2003)] discussed in the following subsections. However, the literature reveals the fact that neither of the strategies is suitable enough to be employed in subsurface. Therefore, an optimal deployment of finite number of sensors in oil fields that are able to communicate efficiently is in high demand.

\subsection{The problem statement: Coverage and Connectivity}

The coverage property [Zou, Y., et al., (2005)] checks that sensors being deployed are adequately covering all data points which are to be sensed or monitored whereas Connectivity checks that these sensors are not placed far apart such that they are unable to communicate with each other. The section discusses various mathematical models employed for efficient coverage and connectivity in various domains.

Researchers [Zhang, H., et al., (2004), Xiaorui Wang, et al., (2003)] proved that for a finite sensor density in $\mathrm{k}$-coverage, $\mathrm{c} \geq 2 \mathrm{r}$ is a necessary and sufficient condition for coverage to imply connectivity where " $\mathrm{r}$ " is sensing range of a sensor or the distance to which a sensor can monitor desired activities, while "c" is the communication range of the sensor. Also, k-coverage with $\mathrm{k}>1$ affords some degree of fault tolerance and is able to monitor all points so long as no more than $\mathrm{k}-1$ sensors fail.

Xu \& Sahni [Xiaochun Xu, et al., (2007)] introduced an Integer Linear Programming (ILP) formulation assuming a set of modalities such as temperature, sound, levels of different gases, radioactivity etc. at each location. This formulation for cost calculation is fine for traditional applications of Wireless Sensors. However, the same cannot be applied directly in a subsurface as it comprises of relatively harsh vibration, temperature and corrosive 
environments. Since, sensor technology is used in a wide range of applications and in various different markets; therefore, these can be deployed in either a phased or iterative manner. This work while making use of pipeline robot developed by Iyengar and his team in the Robotics Research Lab [Iyengar] at Louisiana State University for initially deploying the sensor in area just near well head and activating them to communicate with each other in the first phase, proposes to carry out the second phase of deployment inside subsurface wherein the valuable and critical information governed by the real time factors is inferred with the help of deployed sensors and hence can be utilized for improving the quality and quantity of production. The proposed deployment strategy is discussed in detail in the upcoming section.

\section{The Proposed Deployment Strategy}

The proposed algorithm adopts an incremental deployment strategy that makes use of information provided by previously deployed nodes and is inspired from the works of Andrew et al. [Howard, A., et al., (2002)] who made an attempt to systematize non deterministic deployment, by using the information gathered from previous phase. However, this section proposes a novel deployment algorithm considering the harsh conditions and real-time environment prevailing in subsurface. For instance, in contrast to the author's presumption of homogeneous nodes, this work focuses on heterogeneous nodes. The proposed algorithm aims to maximize both the network coverage as well as the connectivity parameter. Before presenting the algorithm in detail, following are the key considerations that are to be assumed as is for convenience and simplicity.

\subsection{Key Assumptions}

The proposed algorithm has been developed keeping the following three factors as it key assumptions.

- Heterogeneous nodes: The objective is to dynamically guide the sensors to its final location. The increased stability and appropriate location of the sensors helps reducing the number of measurement points required to monitor precisely a surrounding area and consequently reduce the cost of a survey. In order to measure the modalities present within subsurface, various kind of sensor nodes such as temperature sensor, pressure nodes, acoustics nodes, flow nodes etc. will be deployed.

- Geometrical Considerations: It is assumed that the gross topology within the subsurface remains static while the network is being deployed. This implies that the geo obstacles present do not displace during this course of time. The major design challenge that would be simplified i.e. the sensor network be reconfigured, expanded, or otherwise modified without a loss in performance. The static configurations and topologies are conducive to scalability in terms of power and bandwidth usage, congestion control, and overall network robustness to disruptions.

- Mesh Communication: Assuming that each sensor has a sensing radius, " $r$ " and it can only sense the environment and detect events within its sensing area, which is the disk of radius " $r$ " centered at the sensor. A point is said to be covered by a sensor if it is located in the sensing area of the sensor. The sensor network is thus partitioned into two regions, the covered region, which is the region covered by at least one sensor, and the uncovered region, which is the complement of the covered region. A communication is said to be mesh or full if it detects the events happening in both covered and uncovered area. Also, it is assumed that all nodes after being deployed in the network can communicate with remote base-station on which the deployment algorithm is executed.

On the basis of these three assumptions, following section presents a high level view of the proposed algorithm.

\subsection{High Level View}

The algorithm primarily comprises four phases namely, Initialize, Choose, Allocate and Enforce. The flow of information among various phases is as depicted in Fig. 1 and the key functionality of the phases is delineated in Table 1. It may be noted that the nodes are either in waiting, active or deployed state and conditions for each state change is explained with the help of Fig.2.

The algorithm iterates through the Choose, Allocate and Enforce phases, terminating only when all nodes have been deployed. The phases are explained in the following subsections.

1) Initialize

Initialize is the very first phase of the algorithm and initially all nodes are set to waiting state except for one whose state is set to active and is considered as a node ready to be deployed.

2) Choose

This phase determines the next deployment location, or goal and the desired goal is usually the one that either maximizes the coverage metric i.e. $\max ($ coverage_metric) where coverage metric is computed at a location ' 1 ' for 
a modality ' $\mathrm{m}$ ' by maintaining an allocation grid. An Allocation Grid is 2D arrangement of the total area that is likely to be covered. Each cell of the grid is defined as unallocated, allocated or unknown. An unallocated cell implies that it contains no obstacles whereas an allocated cell state implies that it can pose an obstacle. Here obstacle implies a node, which has been deployed in that particular cell. It's being termed as obstacle since it might obstruct the deployment of other nodes to farther cells. In case of non-availability of information about the state of a cell, it is treated as an unknown location i.e. the unknown location cannot be chosen as the next desired destination.

Now, each deployed node communicates its sensing range say ' $r$ ' implying that the upcoming active node shall be deployed in a cell which is not only set as unallocated but also satisfies the relation $c \geq 2 r$, where ' $c$ ' is the communication range ensuring the condition $\max$ (coverage_metric). Depending upon the information gathered, the appropriate cell is chosen and the location of the same is forwarded to the next phase.

3) Allocate

The main aim of the phase is to assign the location chosen during chose phase to a waiting node. The state of the node changes from waiting to active. The phase allocation is simple in case the desired location can be reached unobtrusively. However, if there are nodes already deployed on the path to be followed by the active node, these deployed nodes act as obstruction and such obstructions become increasingly likely to happen as the size of the network increases. This makes

"Allocate" the most important and complex phase of deployment. Now, in order to overcome the stated complexity this work proposes a novel mechanism which allows goal swapping i.e. an active node swaps its own goal with the deployed node leading to the state exchange between two nodes. This implies that the active node acquires the location of deployed node and the deployed node becomes the new active node.

4) Enforce

During this phase, active nodes are ultimately deployed to their goal locations. Node deployment is a consecutive process i.e. only one node remains in active state while all other are either waiting or deployed. Since there is only one node in motion at any given point in time and the goal assignment ensures that each successive goal is unobstructed, there is very little possibility of interference between nodes. However, this deployment process is quite slow. The execution time is proportional to the sum of the distances traveled by the active nodes which in turn is equal to the distance a single node would have traveled if there were no obstructions. As the area covered by the deployed network becomes larger, nodes will have to travel farther and farther and hence it is expected that execution time will increase with the number of deployed nodes.

The upcoming section presents the working algorithm, flowchart of the proposed work, followed by an example of node replacement.

\subsection{Working Algorithm, Flowchart \& Mathematical Model}

4.3.1 Algorithm for the proposed deployment strategy:

Input required for the algorithm:

- $\quad$ node(i): which represents the $i^{\text {th }}$ node to be deployed

- $\quad g_{1}$ : which represents the randomly selected goal where the node(1) is to be deployed.

1) initialize: set status [node(i)] =waiting $\forall i \in\{1,2, \ldots \ldots m\}$

2) set status $[\operatorname{node}(1)]=$ active

3) deploy node(1) at goal $g_{1}$ set status $[\operatorname{node}(1)]=$ deployed

4) repeat

5) collect information from node(i) $\forall i \in\{1,2, \ldots . . m\}$ calculate new goal $\mathrm{g}_{\mathrm{j}} \forall j \in\{2,3, \ldots \ldots m\}$

6) if node(i) $\forall i \in\{1,2, \ldots \ldots m\}$ lie on linear path between point of deployment and $\mathrm{g}_{\mathrm{j}}$ then

7) set status $[\operatorname{node}(\mathrm{i})]=$ active $\forall i \in\{1,2, \ldots \ldots m\}$ redeploy nodes

8) deploy node $(\mathrm{i}+1)$

9) set status[node $(i+1)]$, status[node(i)] = deployed 
10) else

11) deploy node $(\mathrm{i}+1)$ to goal $\mathrm{g}_{\mathrm{j}}$

12) set status[node $(\mathrm{i}+1)]=$ deployed

13) end if

14) until status[node(i)] =deployed $\forall i \in\{1,2, \ldots \ldots . m\}$

15) stop

4.3.2 Flowchart:

Refer to Figure 3 after reference section

\section{An Example}

If $\boldsymbol{n o d e}(\boldsymbol{i})$ obstructs the path to node(i+1) deployment location [Fig 4(a)], then $\boldsymbol{n o d e}(\boldsymbol{i})$ can be redeployed to $\boldsymbol{n o d e}(\boldsymbol{i}+\mathbf{1})$ location, while $\boldsymbol{n o d e}(\boldsymbol{i}+\mathbf{1})$ will occupy the previous location of node(i).

Since all nodes are assumed to be equivalent, this goal-swapping makes no functional difference to the network. For complex environments, with many obstructions, this resolution strategy may need to be applied recursively, so that

$$
\operatorname{node}(\mathbf{j}-1) \sim \operatorname{replaces} \sim \operatorname{node}(\mathbf{j}) \quad \forall j \in i-1, i-2, \ldots \ldots, 1
$$

[Fig 4(b)]

here,

status $\{\operatorname{node}(i)\}=$ active

i.e. it's being deployed in the current iteration

The proposed mechanism executes in an iterative manner oozing the nodes from a central location to an uncovered location. In addition, as the nodes spread throughout the environment, the same nodes will tend to remain on the edge of the network.

\subsubsection{Mathematical Model}

The proposed algorithm with the mentioned assumptions and constraints ( $\mathrm{c} \geq 2 \mathrm{r}$ ) would lead a $2 \mathrm{D}$ allocation grid as shown in Fig.5. The network is formed by placing several horizontal r-strips. The paper [Kar, K., et al., (2003)] defines r-strip as a string of r-disks placed along a line such that the distance between the centers of any two adjacent $r$-disks is $r$. Sensors with a sensing/transmitting radius $r$ can be modeled as a disk with radius $r$. This disk is being referred as an r-disk.

These r-strips are positioned such that the $\mathrm{x}$ and $\mathrm{y}$ co-ordinates of each node are given by:

$$
\begin{aligned}
& x_{i j}= \begin{cases} \pm i r & \text { if } j \text { is odd } \\
\pm \frac{(2 i-1) r}{2} & \text { if } j \text { is even }\end{cases} \\
& y_{i j}=(j-1) \sqrt{15} r / 2
\end{aligned}
$$

,where $\mathrm{i}=0,1,2, \ldots$ and $\mathrm{j}$ refers to the r-strip number $=1,2,3 \ldots$

These r-strips are connected to each other by inserting some more sensors in the network, which will be referred as Global Connectivity Sensors (GCS). The positional co-ordinates of GCS are:

$$
\begin{gathered}
\mathrm{x}_{\mathrm{i}}=(-1)^{\mathrm{i}} \mathrm{r} / 4 \\
y_{i}=(2 i+1) \sqrt{15} r / 4
\end{gathered}
$$

where $\mathrm{i}=0,1,2, \ldots$.

Let us consider a portion of the network consisting of the sensors 1-6, as shown in Fig.6. The dots $(\bullet)$ represent deployed sensors nodes. The sensors 1 and 3 are located within two odd-numbered r-strips, whereas, the sensors 2 and 4 are within an even-numbered r-strip. The GCS (sensors 5 and 6) are positioned so as to connect the sensor 1 to 4 and 2 to 3 and hence, the corresponding r-strips are connected to each other. Such a placement of GCS allows an increase in the vertical spacing between the r-strips as compared to the network described by Kar and Banerjee [Kar, K., et al., (2003)]. This eliminates any overlapping between the r-strips (See Fig. 6) and hence, an increase in the coverage area as compared to [Kar, K., et al., (2003)], the details of which are presented in the next subsection. 


\subsubsection{Performance Evaluation and Comparison}

As per the paper [Kar, K., et al., (2003)], nodes of adjacent r-strips are inter-connected with each other by inserting new nodes in between them (Node E \& F in this case). Here node A \& C lies on odd r-strips, while node B \& D lies on even r-strips.

To ensure connectivity, maximum permissible distance between two deployed nodes $(B \& D)=r$. Sensor E \& F had to be so placed that their distance from A, B, C, D = r.

Since distance between node B and node D is equal to $\mathrm{r}$, nodes $\mathrm{E} \& \mathrm{~F}$ are placed parallel to nodes A \& $\mathrm{C}$. Fig 7 . Therefore

$$
\mathrm{BO}=\mathrm{OD}=\mathrm{r} / 2 .
$$

Now let's compute $\mathrm{EO}$ from right angled triangle $\mathrm{BOE}$,

$$
\begin{gathered}
\mathrm{BE}^{2}=\mathrm{BO}^{2}+\mathrm{EO}^{2} \\
\mathrm{r}^{2}=(\mathrm{r} / 2)^{2}+\mathrm{EO}^{2} \\
\mathrm{EO}=\sqrt{\left(\mathrm{r}^{2}-\mathrm{r}^{2} / 4\right)=\sqrt{3 r} / 2}
\end{gathered}
$$

This gives,

$$
\begin{aligned}
& \mathrm{AO}=\mathrm{AE}+\mathrm{EO} \\
& \begin{aligned}
=\mathrm{r} & +\sqrt{3} r / 2 \\
= & 1.866 \mathrm{r}
\end{aligned}
\end{aligned}
$$

which, basically is the vertical spacing between two r-strips as proposed by [Kar, K., et al., (2003)]. Similarly, compute $\mathrm{AB}$ from right angled triangle $\mathrm{AOB}$,

$$
\begin{gathered}
\mathrm{AB}=\sqrt{ } \mathrm{BO}^{2}+\mathrm{AO}^{2} \\
=\sqrt{ }(\mathrm{r} / 2)^{2}+(\mathrm{r}+(\sqrt{ } 3 / 2) \mathrm{r})^{2} \\
=1.931 \mathrm{r}<2 \mathrm{r} .
\end{gathered}
$$

Hence there is overlapping between r-disks lying on alternate r-strips. This is the limitation of previous work. In order to overcome this limitation, we propose to increase vertical spacing so as to reduce overlapping. This can be done by inserting the interconnecting nodes $\mathrm{E} \& \mathrm{~F}$ at points as specified in Fig. 8. With such placement, we would have

$$
\mathrm{BO}=\mathrm{OD}=\mathrm{r} / 2
$$

and

$$
\mathrm{AB}=2 \mathrm{r}
$$

Now let's compute the vertical distance (AO) between alternate r-strips,

$$
\begin{gathered}
\mathrm{AO}=\sqrt{ } \mathrm{AB}^{2}-\mathrm{BO}^{2} \\
=\sqrt{ }(2 \mathrm{r})^{2}-\mathrm{r}^{2} / 4 \\
=\sqrt{15 / 2 \mathrm{r}}=1.936 \mathrm{r}
\end{gathered}
$$

The value of AO reflects that our algorithm has been able to increase the vertical spacing between two r-strips which in turn would lead to reduced overlapping and also the number of sensors to be deployed. It implies that overall time of deployment of sensors, the cost of deployment and the cost of sensors is significantly reduced. The result clearly indicates that our algorithm and placement pattern provides connected coverage to entire field.

\section{Conclusions and Future Work}

The work presented in this paper makes a unique contribution towards the deployment of wireless sensors in subsurface as at the time of listing, none other researchers have proposed any mechanism to deploy sensors in subsurface and also the proposed strategy of deployment is efficient as compared to other existing strategies in other related fields. The work only focused on the deployment of sensors ignoring the communication strategy or routing protocols to be employed. A routing protocol that can adapt to the prevailing conditions, node failure and data loses shall be taken over as future work. 


\section{References}

AEC. (2008). [Online]. Oil and Gas Micro-and Nano-sensor Needs. Advanced Energy Consortium. Retrieved from http://www.beg.utexas.edu/aec/pdf/AEC_Sensor\%20Needs_072008.pdf

Akkaya, K. ., \& Younis, M.. (2005). A Survey of Routing Protocols in Wireless Sensor Networks. Elsevier Ad Hoc Network Journal. 3/3. 325-349.

Alan Mainwaring, Joseph Polastre, Robert Szewczyk, David Culler \& John Anderson. (2002). Wireless Sensor Networks for Habitat Monitoring. ACM WSNA'02, September 28, 2002, Atlanta, Georgia, USA. 88-97.

B. Liu, P. Brass, O. Dousse. (2005). Mobility Improves Coverage of Sensor Networks. Proc. of the the 6th ACM Intl. Symposium on Mobile Ad Hoc Networking \& Computing (MobiHoc '05), Urbana-Champaign, Illinois, USA. 300-308.

Bai, X. , Kumar, S. , Yun, Z. , Xuan, D. \& Lai, T. H.. (2006). Deploying wireless sensors to achieve both coverage and connectivity. ACM International Symposium on Mobile Ad Hoc Networking and Computing, Florence, Italy, 2006. 131-142.

Bin Lu, Thomas G. Habetler, Ronald G. Harley \& José A. Gutiérrez. (2005). Applying Wireless Sensor Networks in Industrial Plant Energy Management Systems - Part I: A Closed-Loop Scheme. Sensors, 2005 IEEE, Oct. 30 2005-Nov. 3 2005. 145-150.

CIA. Central Intelligence Authority Fact $\quad$ Sheets. [Online] https://www.cia.gov/library/publications/the-world-factbook/geos/us.html.

CNBC. [Online] http://www.cnbc.com/id/25123762.

Colibrys. [Online] www.colibrys.com

David Culler, Deborah Estrin \& Mani Srivastava. (2004). Overview of Sensor Networks. IEEE Computer Society, August 2004. 41-49

Eisenman, S. B. , Lane, N. D. , Miluzzo, E. . Peterson, R.A. , Ahn, G. , \& Campbell, A.T.. (2006). Metrosense project: People-centric sensing at scale. First Workshop on World-Sensor-Web (WSW'2006), SenSys'06, Oct, 2006.

Estrin, D. , Girod, L. , Pottie, G. , Srivastava, M.. (2001). Instrumenting the world with wireless sensor networks. International Conference on Acoustics, Speech, and Signal Processing (ICASSP 2001), Salt Lake City, Utah, May 2001.

Estrin, D. , Govindan, R. , Heidemann, J. \& Kumar, S. (1999). Next century challenges: scalable coordination in sensor networks. ACM MobiCom'99, Washingtion, USA, 1999. 263-270.

Ghosh, A. (2004). Estimating coverage holes and enhancing coverage in mixed sensor networks. IEEE Int. Conf. Local Computer Networks, Nov. 2004. 68-76.

Howard, A. , Mataric, M. \& Sukhatme, G.. (2002). An incremental self-deployment algorithm for mobile sensor networks. Autonomous Robots, Special Issue on Intelligent Embedded Systems. 13(2), Sept 2002. 113-126.

Huang, C.-F. \& Tseng, Y.C.. (2003). The coverage problem in a wireless sensor network. ACM International Workshop on Wireless Sensor Networks and Applications (WSNA), 2003. 115-121.

Iyengar. [Online] http://csc.lsu.edu/ iyengar/.

Juneja, Dimple, Sharma, Atul. \& Sharma, A.K. (2009). On the role of Wireless Sensor Networks in Subsurface Exploration: An Overview. IEEE International Advance Computing Conference' 2009 (IACC), ISBN 978-981-08-2465-5.

Kar, K. \& Banerjee, S. (2003). Node Placement for Connected Coverage in Sensor Networks. Extended Abstract. Proceedings of 1st Intl. Symposium on Modeling and Optimization in Mobile, Ad-hoc and Wireless Networks (WiOpt), Sophia-Antipolis, France, March 2003.

Nils Timotheus Kannengießer \& Helmut Dispert. (2008). Implementation of a Security System based on RFID and WSN Technology. [Online] TechRepublic Whitepapers, Sep 2008 http://whitepapers.techrepublic.com.com/abstract.aspx?docid=961375.

Roger M. Slatt, Thomasson, M. Ray., Philip R. Romig, Eric S. Pasternack, Albert Boulanger, Roger N. Anderson, $\&$ H. Roice Nelson. (2004). Visualization technology for the oil and gas industry; today and tomorrow. AAPG Bulletin; April 1996. v. 80; no. 4. 453-459. 
Sammarco, J.J., Paddock, R. , Edward F. Fries, Vijia K. Karra. (2007). A Technology Review of Smart Sensors With Wireless Networks for Applications in Hazardous Work Environments. IC 9496, Information Circular 2007.

Sohrabi, K. , Gao, J. , Ailawadhi, V. , Pottie, G.J. (2000). Protocols for self-organization of a wireless sensor network. IEEE Personal Communications, October 2000. 16-27

Tatiana Bokareva, Wen Hu, Salil Kanhere, Branko Ristic, Neil Gordon, Travis Bessell, Mark Rutten \& Sanjay Jha. (2006). Wireless Sensor Networks for Battlefield Surveillance. Land Warfare Conference 2006, Brisbane, Australia, October 2006.

Virone, G. , Wood, A. , Selavo, L. , Cao, Q. , Fang, L. , Doan, T. , He, Z. \& Stankovic, J.A.. (2006). An Advanced Wireless Sensor Network for Health Monitoring. Transdisciplinary Conference on Distributed Diagnosis and Home Healthcare (D2H2), Arlington, VA, April 2-4, 2006.

Wang, X. , Xing, G. , Zhang, Y. , Lu, C. , Pless, R. \& Gill, C.. (2003). Integrated coverage and connectivity configuration in wireless sensor networks. First Int. Conf. Embedded Netw. Sensor Syst., Nov. 2003. 28-39.

Wang, Y.C. , Hu, C.C. \& Tseng, Y.C.. (2005). Efficient deployment algorithms for ensuring coverage and connectivity of wireless sensor networks. Proc. IEEE Wireless Internet Conf.. 114-121.

WSN. Wireless Sensor Networks. [Online] http://en.wikipedia.org/wiki/Wireless_Sensor_Networks.

X. Du \& F. Lin. (2005). Improving sensor network performance by deploying mobile sensors. Proc. IEEE Int'l Performance, Computing, and Comm. Conf., 67-71.

Xiaochun Xu \& Sartaj Sahni. (2007). Approximation Algorithms For Wireless Sensor Deployment. IEEE Transactions on Computers. vol.56, no.12. 1681-1695.

Xiaorui Wang, Guoliang Xing, Yuanfang Zhang, Chenyang Lu, Robert Pless, \& Christopher Gill. (2003). Integrated Coverage and Connectivity Configuration in Wireless Sensor Networks. ACM SenSys, November 2003. 28-39.

Zhang, H. \& Hou, J. C. (2004). Maintaining sensing coverage and connectivity in large sensor networks. NSF International Workshop on Theoretical and Algorithmic Aspects of Sensor, Ad Hoc Wireless, and Peer-to-Peer Networks. 89-124.

Zou, Y. , \& Chakrabarty, K. (2005). A Distributed Coverage- and Connectivity-Centric Technique for Selecting Active Nodes in Wireless Sensor Networks. IEEE Transactions on Computers. Vol. 54, No. 8. 978-991.

Table 1. Phases and their Key Functionalities

\begin{tabular}{|l|l|}
\hline Phases & Key functionality \\
\hline Initialize & Set the state of a node to waiting, active or deployed. \\
\hline Choose & $\begin{array}{l}\text { Develop a location map with the help of deployed nodes and select the appropriate location for } \\
\text { waiting node. }\end{array}$ \\
\hline Allocate & Assign destination to a waiting node and set the state as active. \\
\hline Enforce & Active node is deployed at the desired location successively \\
\hline
\end{tabular}




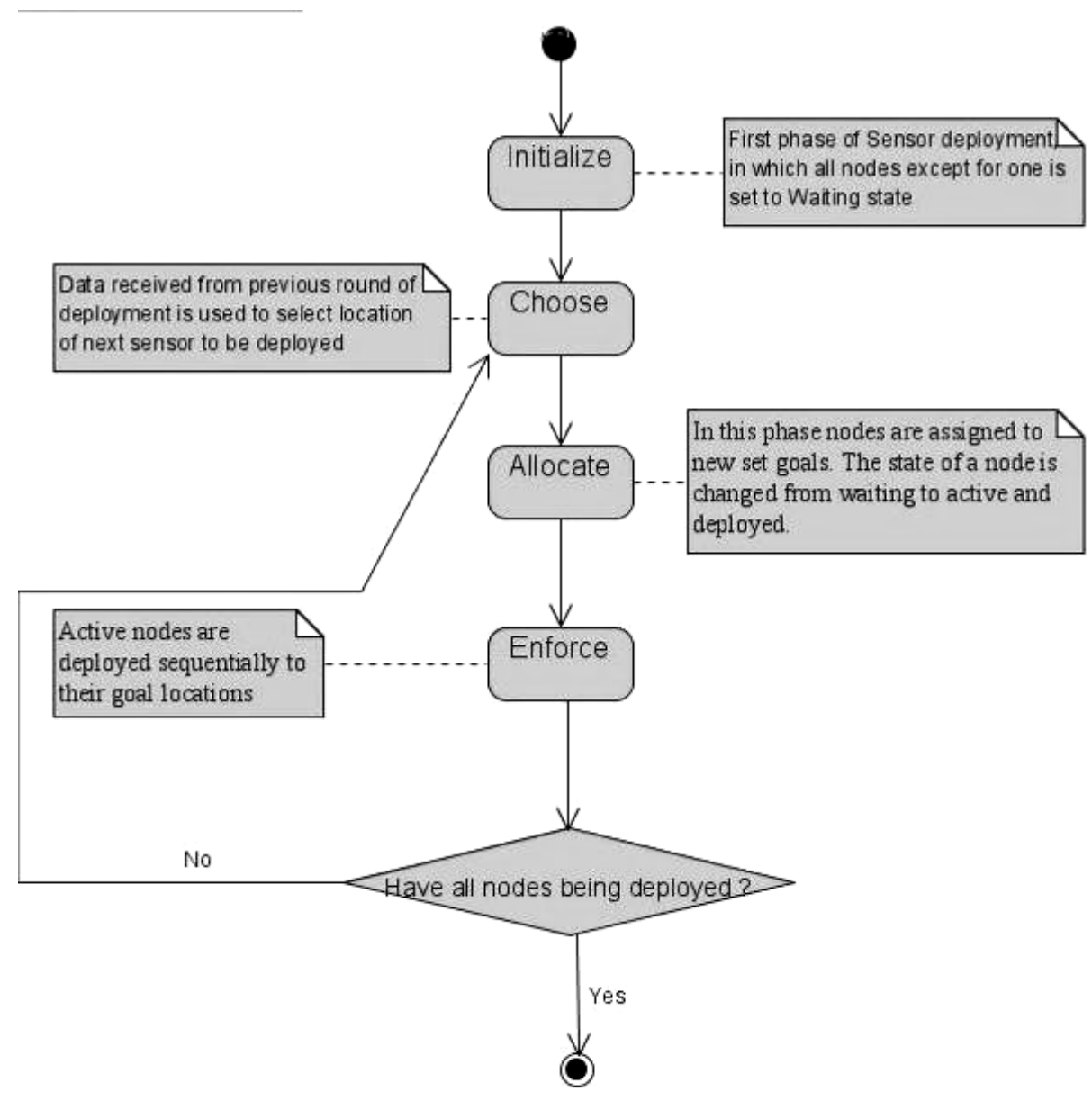

Figure 1. Transition between various phases of the algorithm

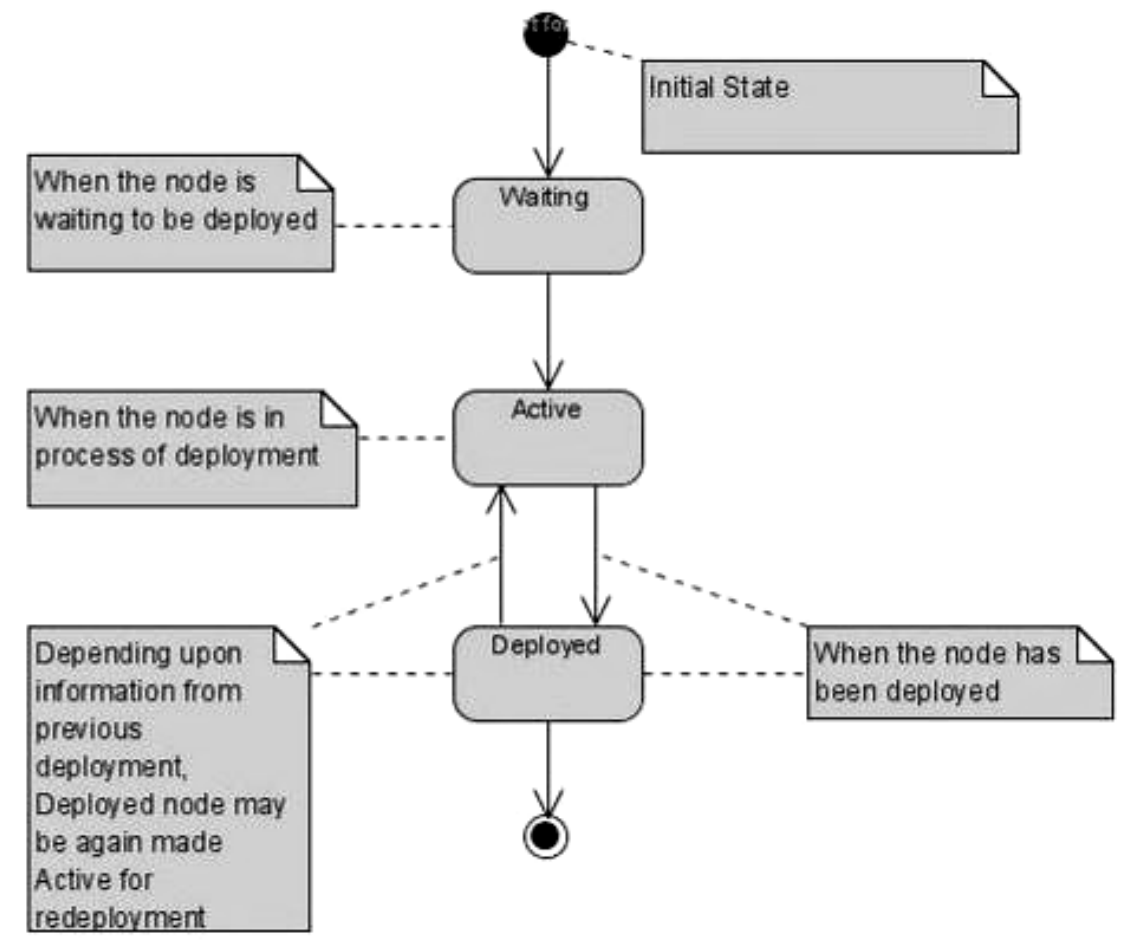

Figure 2. State Diagram of nodes being deployed 


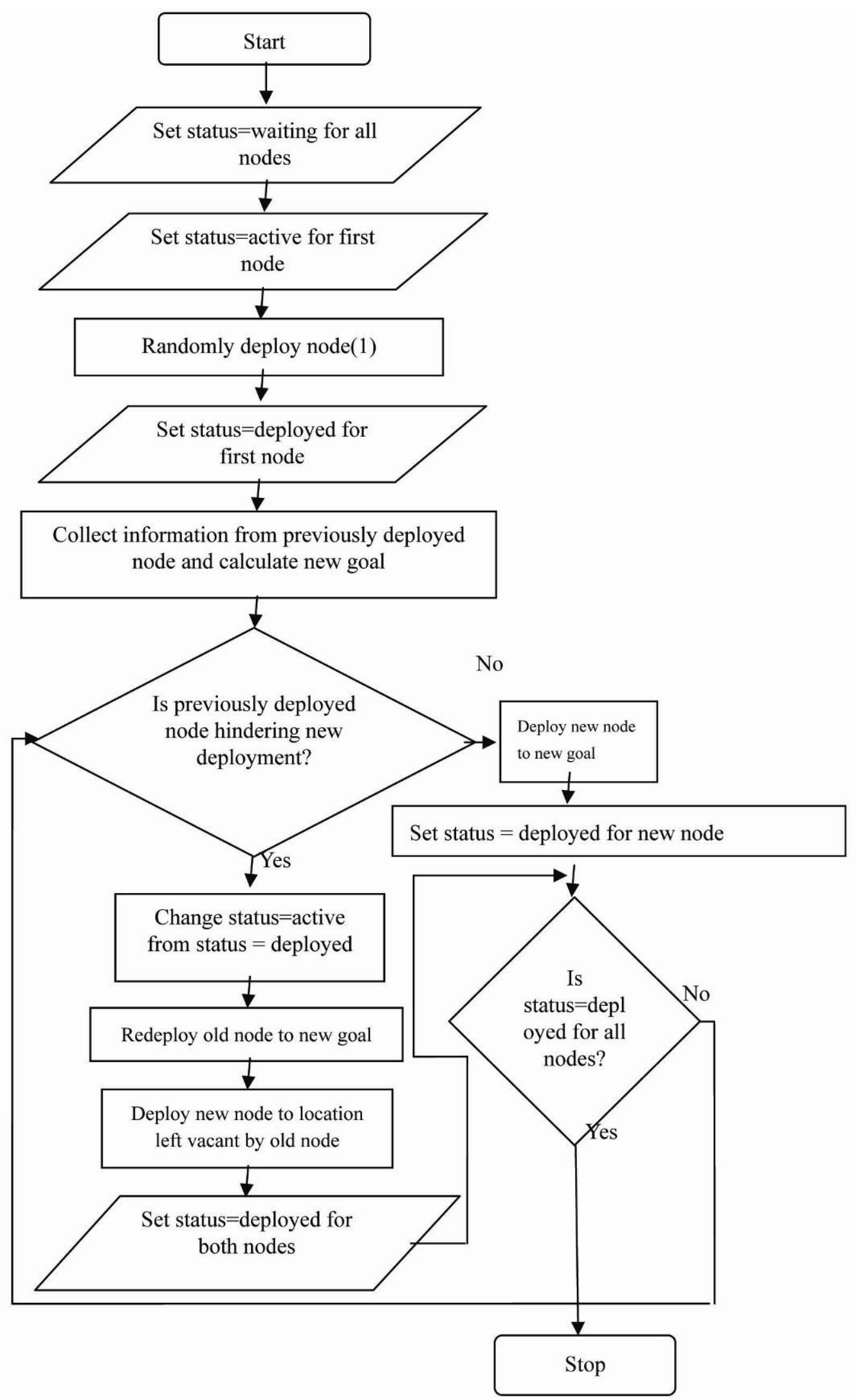

Figure 3. Flowchart 
(a)
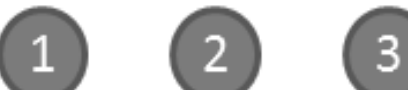

Desired Location for Node 4

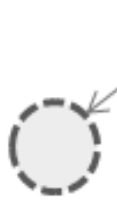

(b)

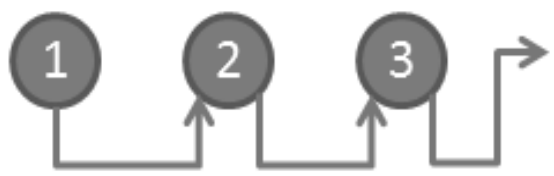

Node 1 moves to location of Node 2, Node 2

to Node 3 , and Node 3 to desired location for Node 4

(c)

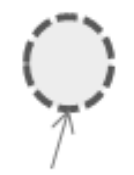

Location for Node 4 to be deployed now

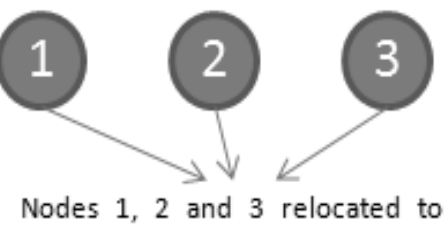
new goals

Figure 4. Iterative redeployment of nodes

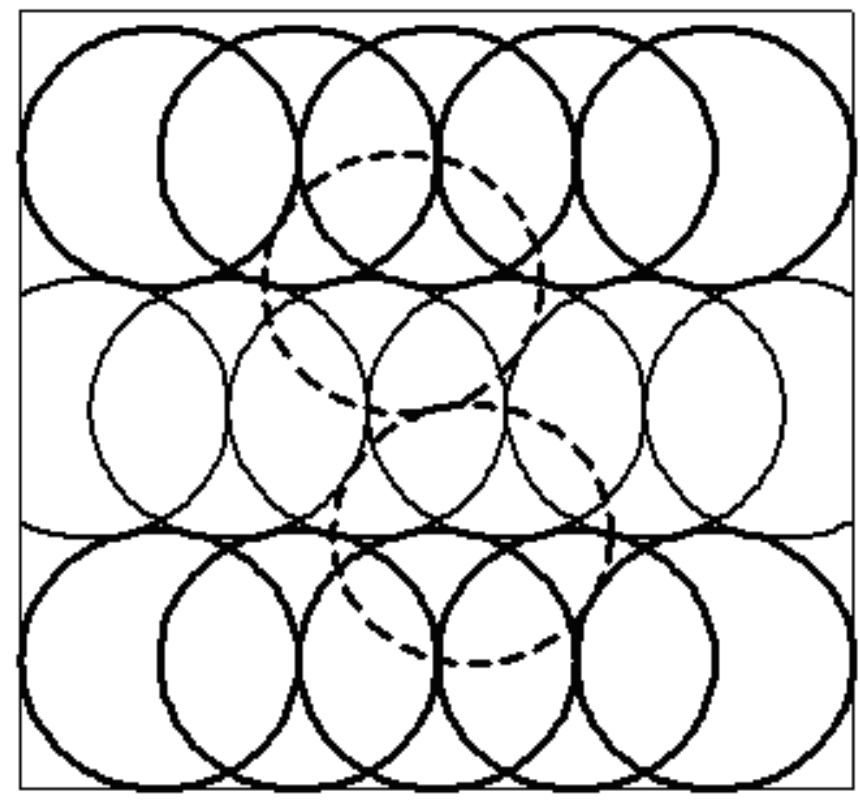

Figure 5. A 2D Allocation Grid 


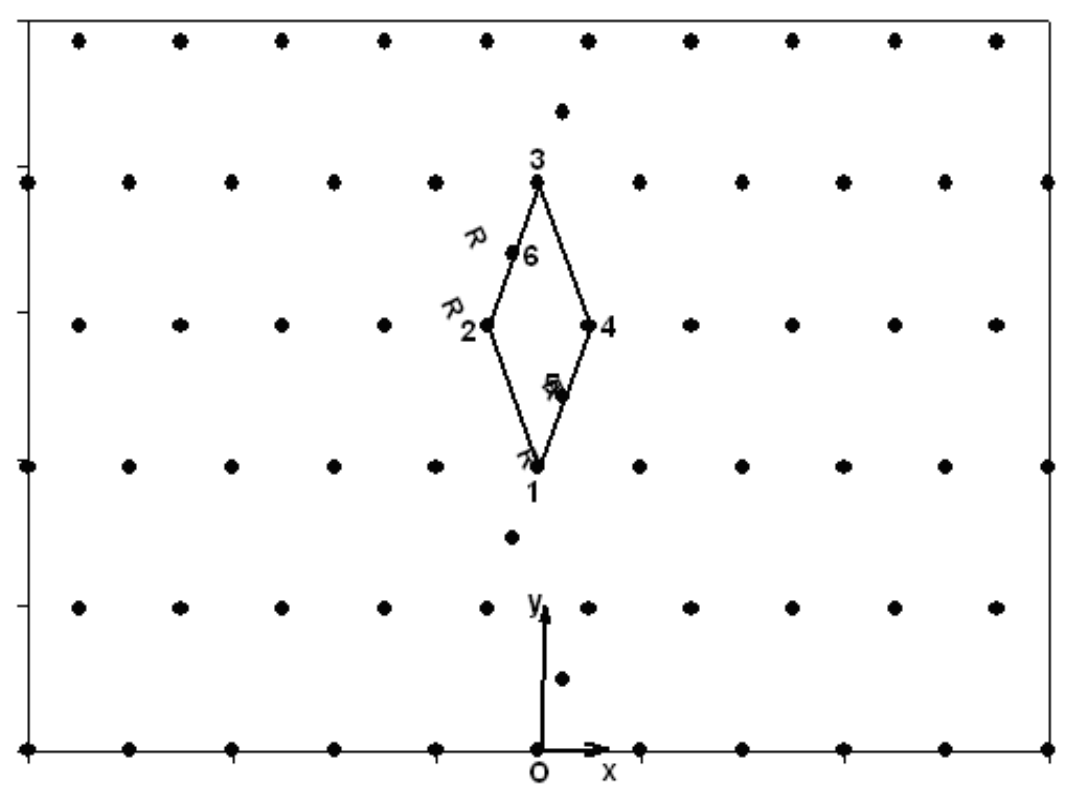

Figure 6. Connectivity and Coverage Description

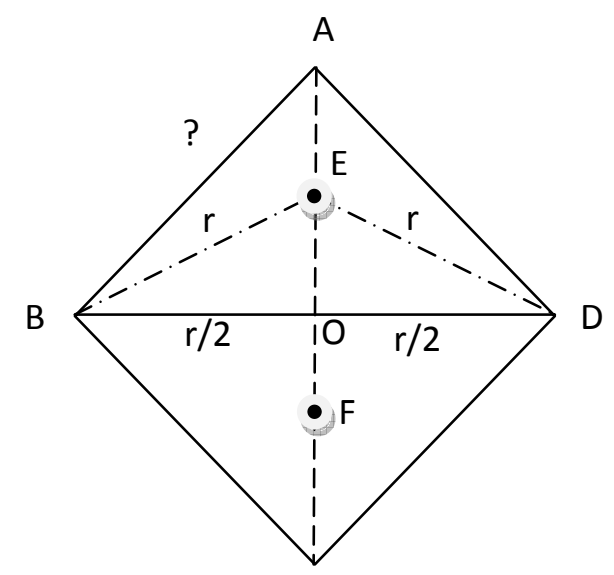

C

Figure 7. Detailed view of r-strips placement as per [Kar, K., et al., (2003)]

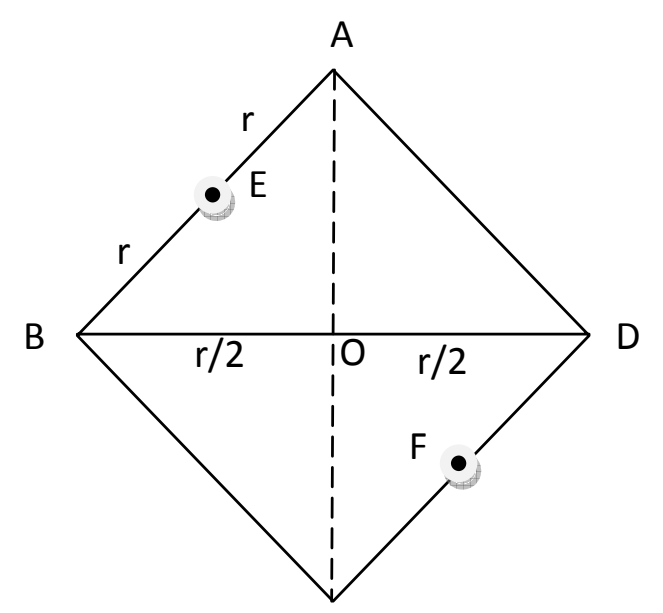

C

Figure 8. Detailed view of r-strips placement as per our proposed model 